AI \& Soc (1991) 5: 239-254

\title{
Open Forum
}

\section{Virtuality as a Basis for Problem Solving?}

\author{
Gianni Degli Antoni and Rita Pizzi \\ Università degli Studi di Milano, Dipartimento di Scienze della Informazione, 20133 Milano, Italy
}

\begin{abstract}
This paper is an attempt to develop a paradigm in problem solving where the notion of virtuality plays a central role. Following a brief discussion on virtual simulation, the paper attempts to identify a notion of virtuality based on the identification of the self (man or computer) with the problem solver. It is shown that such an identification/ distinction possibly violates some general principle of the problem environment.

To deal with this violation, a new problem is generated and a new virtualization act is required, in order to attain a correspondence between virtuality and reality. A better understanding of this approach requires a combination of analog and digital reasoning: the analog being related to the environment in which the problem solving is required, and the digital to the problem solver's "mental" activities.

We will briefly analyze the recursive approach to problem solving and suggest a possible problem solving methodology based on virtuality. The paper concludes with comments on the relation between etnology and virtual reality.
\end{abstract}

Keywords: Problem solving; Virtual reality

\section{Introduction}

Recent approaches in artificial realities and virtual environments combine technology with man's creativity. This paper aims to investigate the underlying ideas of these approaches, and argue that virtuality is a basis for human problem solving and for building a general problem solving paradigm which can be used in computer based environments. The discussion will identify the basic concepts that we can use to improve our notion of virtuality. 


\section{From Virtual Reality to Problem Solving}

Much attention has recently been paid to the virtual or artificial reality approaches for creating computer-based environments, where the user perceives virtual or real entities and acts as an agent in direct contact with those entities (Krueger, 1983).

Various degrees of fiction are possible, depending on the technology used to give perception of the environment, ranging from graphical 2-dimensional displays (where the mouse pointer is to be considered as the user acting on objects (Degli Antoni, 1988)) up to 3-dimensional full-immersion displays endowed with electronic clothing which inserts the user's body in the computer environment (Fisher, 1986).

Artificial reality has generated interest in many applications where simple graphics or sophisticated perception equipments are needed (Minsky, 1984). Applications range from office and CAD systems to entertainment devices (Moshell, 1990). All these systems take advantage of the cognitive content of the computer interfaces, in the case of obvious standard user interfaces or in the sophisticated illusions made possible by the input/output devices. The essence of the cognitive content is contained in the interface between physical nature of the entities and a suitable analog language (Schneiderman, 1983).

The analog language enables the user to perceive well-known entities belonging to an eventually unknown world, and thereby simplifies the computer-user interaction. The user thus perceives cognitive information systems in terms of the subject matter only without the need of any knowledge of computer technology. The approach has been useful even in many non-trivial fields, for example, in representing the knowledge of the behaviour of HIV Viruses in their interaction with the immunologic system (Di Floriano, 1990).

If the best way to understand conceptual entities is through metaphors that display those entities in a space, then it is likely that future applications of artificial or virtual realities will be just the invention of conceptual real or virtual spaces where the user will have the possibility of navigating and solving new problems (Farmer, 1990). Very interesting examples of this possibility can be taken from various fields of mathematics and computer sciences, physics, operation research and many other disciplines. Graphs, trees, Venn diagrams, semantic nets, Petri nets, connectionism entities, arrows, functors, state machines, Feynman graphs, transportation graphs, biochemical nets, bond graphs in mechanics and thermodynamics are some of the most known results of translating concepts in a physical picture. This continues the tradition of geometry not only in representing objects, but also in using deductive approaches, as Euclid did two thousand years ago. The similarity between geometrical concepts and natural entities has certainly been important for the diffusion of geometry.

A similar naive approach is spreading in the field of other representation techniques. This naive approach is becoming questionable if less intuitive representations, as those developed for fractal geometry, can give pictures of the real world better than those implied by a naive Euclid geometry.

If one takes into account the above issues, then science appears related to the representation of spaces of known or partially unknown entities, the navigation 
tools in those spaces, and the use of maintenance tools to integrate new discoveries of the conceptual virtual or real spaces. But how to make new discoveries, how to extend our notions, how to make user or computer more intelligent? Can navigating in conceptual virtual or artificial spaces increase the understanding of the human being?

We will leave all these questions open. We will only try to investigate here if the idea of virtuality can be used to build a new paradigm of problem solving that we hope could overcome the current impasse of artificial intelligence in building truly intelligent agents. The idea of virtuality is not new in the computing field: virtual registers, virtual stores, virtual discs, virtual machines, virtual channels, virtual interfaces, virtual keyboards, virtual terminals, virtual networks and recently even virtual users, have been proposed and extensively used in the field of computing technology.

In all these applications, by "virtual" we mean entities that do not exist, but under suitable operational conditions behave as if they were real entities. Thus for example a virtual machine in a given computer behaves like the prototype used to implement the virtual machine itself. Even without a deeper analysis of the term "virtual", it is clear that a virtual entity has certain useful properties required under suitable hypotheses that could violate basic principles. In this sense, all virtual realities introduced so far appear to be useful: for example, the user can be plunged in a virtual space even a hundred miles away. The example of the virtual machine violates some rule of existence and makes existing a nonexisting machine, under a suitable well behaving of a real computer.

It is not our intention to go into the virtuality issue: nevertheless a result seems to emerge from our virtual construction that certainly (as any virtual system does) violates some basic well definite principles. The result (a virtual one) is the following: we can apply the idea of virtuality to problem solving. We have only to go recursively in greater detail just to the point where we are able to solve the problem. In the next sections we will try to develop further this idea.

\section{Virtuality as a Paradigm of Problem Solving}

Virtual environments can usefully be used in human problem solving. Various approaches can be considered, all share the need of an environment where the problem models perceivable by the user can be directly manipulated.

An example of the use of virtual environments in problem solving is simulation. The user has the possibility of observing directly the behaviour of the system using his conceptualization tools without making reference to data representation techniques or languages. He perceives the system directly and has some control over the system behaviour or structure depending on the flexibility of the system itself.

A well-designed system will allow a change of the structure with the least possible changes in the choices of behaviour. The user will have direct capabilities of judgement with respect to the nature of the problem. Abstractions will be made by selecting an observation "distance" from the virtual reality represented by the simulated system. The user can also modify structure and behaviour 
parameters for a better understanding. Moreover, he can use virtual tools to construct observation or computations as close as possible to his own perceptions. The problem solutions will be represented by a choice of parameters and/or structures verifying the requirements of the problem. This approach is followed in many real-world applications, but it is inadequate where problem solving is to be performed directly by the machine.

\section{Problem Solving and Virtuality}

Many paradigms have been considered in problem solving: some require a deductive capability, others are based on genetic trials where many agents use different approaches to solve (part of) the same problem (Newell, 1972).

Learning, genetic or not, is considered more and more a general purpose problem solving approach which requires examples and not a logical description of the problem solving process (Carbonell, 1983). Some approaches to learning lead directly to subsymbolic solutions, as in the case of neural networks.

Understanding, if a neural network has correctly learnt, is a problem probably more difficult than that of solving it symbolically through deductive techniques based on suitable representations of the problem details. Thus, communicating the content of the solution of a problem through neural networks is a difficult task where symbolic and subsymbolic approaches are combined.

Artificial intelligence tries to build systems which perform general problem solving abilities. Building upon the pioneering work of Newell and others with GPS (General Problem Solver) (Ernst and Newell, 1969), the general problem solving idea has been transformed into automatic programming whereby, given the representation of the problem specification in a suitable language, the computer solves the problem without any human intervention.

Declarative languages such as PROLOG include features and representation indicated by the early attempt in general problem solving (Bratko, 1986). Prolog suffers from limitations related to the use of the Horn clauses and to the low performances if used merely in a purely declarative way. To overcome these difficulties of representation, the problem description has to be mixed with partial descriptions of the problem solution. Unfortunately this happens even in simple cases where a purely declarative style could be desirable, for example in the wellknown monkey-banana problem where a modest model of intelligence is implied (Green, 1969; Lee, 1986; Nilsson, 1976).

The problem solving paradigms implicitly embody some suggestions from virtuality: for simplicity it is enough to consider the backtracking techniques. During the backtracking, the solver (the machine) becomes a virtual copy of itself, trying a different way to the solution in a new virtual world. If something useful is found in this new world, then the main solver is informed.

Virtuality could become a basic paradigm for general problem solving. In this paradigm, the user becomes the virtual problem solver. He can imagine the situations which can solve the problem even if these violate some constraints, dependent on the availability of tools and on the nature of the environment in 
which the user operates. Then the problem solver should be able to build, in the imaginated world, constructions that enable him to overcome the difficulties.

To clarify this approach, let us consider the case of the monkey-banana problem. The monkey looks at the bananas on the ceiling. He wants bananas and immediately realizes the problem: bananas are not reachable by jumping. The monkey is able to reach bananas only through the imagination: he can imagine himself to be near the bananas or tall enough to reach them. The imaginated solutions are set in a virtual world, i.e. a copy of the real world that the monkey has modelled in his mind. But the monkey knows that by stretching, he can obtain only limited results, thus he must exclude this solution and analyze the other one. $\mathrm{He}$ understands that, in order to be near bananas, he has to violate the gravity. However, he knows through experience that, if some tool is available to reach that level, it is possible to stay in that position. Now the problem has fully changed: it is no longer to reach bananas, but rather to reach the position where bananas are reachable, and to stay there long enough to grasp bananas. This new problem has now to be solved, searching in the real space. Here he finds a box and virtually imagines the solution: the box under the bananas, himself upon the box.

So the problem is to implement the solution only: virtually imagined states must be substituted by real states, in an order compatible with the physical constraints. Thus the box will be displaced, the monkey will climb the box and will grasp bananas. Simulated solutions of this approach are fully feasible and do not present the difficulties of the approaches mentioned above (Degli Antoni and Pizzi, 1991).

This example deserves some observations. First of all it is noted that the solution of the problem is built up analogically in a virtual (or imaginated) world. This is essentially an analog world, in a twofold sense: similar but not identical, and not digital. The world is built up by eliminating the gravity, the peculiarity that makes impossible the solution of the problem in the usual world. An interesting item which seems to capture elements of the idea of virtuality is the instrumental use that the problem solver makes of the virtual self: firstly he becomes a displacer (of the box), then a jumper: an instrument of the environment, totally dependent on the action he is taking over the selected object. The user is only giving virtual life to the selected object. Actually, the user is the object of the virtual reasoning. The object behaves as a consequence of the virtual transformation performed by the problem solver. At the end of this plan the execution is made by transforming virtual situations into real ones, backtracking if something is wrong. At the end of this analysis, it is to be noted that if one considers the box over which the monkey climbs as an extension of the monkey (prothesis), then the monkey can be considered a virtual monkey where the implementation of virtuality has given the monkey the possibility to reach the bananas himself.

Transforming the above discussion into a general approach to problem solving is quite exciting. A first rough trial has been presented at the First Conference on Cyberspace, where the Spencer-Brown's logic has been used to understand some aspects of artificial reality (Pizzi and Degli Antoni, 1990). A further investigation is needed to develop such a problem solving approach based on the role of imagination. Nevertheless, the discussion has illustrated that the integration of 
the analog virtualization of the problem solver with the logical approach could lead to a new approach. In particular, the virtualization seems to be related to the recursive definitions (Pizzi, 1990). In fact virtual transformations are implied even in the recursive definitions; but the transformation can not be without knowledge of what happens when the definition is applied.

The advantage of our approach compared to the standard one is that, while traditional recursion gives only a name to its next step, our proposal makes the solution explicit by means of a virtual transformation. In some sense this suggests an underlying process to be followed in writing a recursive definition.

It seems likely that the sequence of virtualization undergoing the problem solving process can be managed by self-refiexive languages.

\section{Beyond Problem Solving: A Look at the Man}

Nowadays we have many ideas about problem solving. In the remote history of man, understanding problem solving has not been an easy task, and even now the efforts of computer scientists in this direction have not been fully resolved.

Historically, symbols were discovered as a medium for problem solving and the communication between the others and the self. Particularly relevant to this discussion is the discovery of the alphabet. Carraro (1987) points out that the alphabet signs were created in many independent cultures by the transfiguration of the human body. We can incorporate this transfiguration into our metaphor: this appears to imply that virtuality has to be considered as a reasoning tool in etnology as well as in the description of human behaviour, and not only in the field of artificial problem solving.

This relation of human behaviour to design is particularly remarkable. Design is the direct consequence of imagination. Imagination is the result of the virtualization of the user with respect to problem solving. The circular relation between imagination and design is certainly based on the obsession of man to modify his own world (Nilsson, 1971).

With the advent of artificial reality, we enter a higher order of problem solving, where we can distinguish or confuse the subject from the object as never in the history of man: this distinction/coincidence is just a magic result of virtualization. When the act of virtualization fails, then a true man appears again with his immense amount of history and problems. It is probably for these reasons that the artificial worlds will simplify and at the same time complicate our world.

\section{Acknowledgements}

It is difficult to recognize the many contributions that have led to the above ideas. The Hypertext User Group (HUG) has been the main place of discussions and comparison where many experimental prototypes have been designed and analyzed and have implicitly suggested many of the ideas of this paper.

Nevertheless, some people must be mentioned: Francesco Gardin, who pioneered visions of navigating in complex virtual realities; Bernard Meltzer, for his critical and constructive attitude with respect to our attempt to understand 
virtuality in problem solving; Gualtiero and Roberto Carraro for their creativity about artificial worlds and their deep metaphors and observations. Without the criticism and suggestions of Roberta Macchi this paper would have never appeared.

\section{References}

Bratko, I. (1986). Prolog Programming for Artificial Intelligence, Addison-Wesley.

Carbonell, J. G. (1983). Learning by analogy formulating and generalizing plans from past experience. In ed. Michalski, R. S. Carbonell, J. G. Mitchell, T. M. Machine Learning. Tioga Publishing Company.

Carraro, G. and R. (1987). Letterografia: l'alfabeto in immagini, F. Mott ed., April 1987.

Degli, Antoni G. (1988). Artificial Worlds. Proc. of the II Conference of Hypertext User Group, Milan.

Degli, Antoni G. (1990). Realta' Artificiale: Una Silenziosa Rivoluzione Cognitiva. Internal Report $81 / 90$, Department of Information Sciences, University of Milan.

Degli, Antoni G. and Pizzi, R. (1991). Virtuality as a Paradigm of Problem Solving. II Conference on Cyberspace, Santa Cruz (submitted).

Di Floriano, M. (1990). Browsing in knowledge through animation: an application to the aids case. Note di Software 48/49, June/ October 1990.

Ernst, G. W. and Newell, A. (1969). GPS: A Case Study in Generality and Problem Solving. ACM Monograph, Academic Press Inc., New York.

Farmer, F. R. (1990). Cyberspace: Getting there from Here. Proc. of the First Conference on Cyberspace, Austin.

Fisher, S. S. (1986). Telepresence Master Glove Controller for Dexterous robotic End-Effectors. Proc. SPIE, vol. 76, "Intelligent Robots and Computer Vision".

Green, C. (1969). Application of Theorem Proving to Problem Solving. Proceedings of IJCAI.

Krueger, M. (1983), Artificial Reality, Addison-Wesley.

Lee, G. F. (1986), Tutorial on Robotics. 2nd ed., Computer Society Press

Minsky M. R. (1984). Manipulating simulated objects with real-world gestures using force and position sensitive screen. ACM Computer Graphics, vol. 18, n. 3, July.

Moshell, J. M. (1990). Simnet and Ender's Game: Military Training in Cyberspace. Proc. of the First Conference on Cyberspace, Austin.

Newell, A., Simon H. A. and Shaw G. L. (1972). Human Problem Solving, Prentice-Hall Inc., New Jersey.

Nilsson, N. J. (1971). Problem Solving Methods in a, McGraw Hill.

Nilsson, N. J. (1976). Methods in Resolution of Problems in Artificial Intelligence. F. Angeli.

Pizzi, R. (1990). Self-Observation in Artificial Reality. Note di Software 48/49, June/October.

Pizzi, R. and Degli Antoni, G. (1990). An Introductory Model of Artificial Reality: Relationship with Spencer-Brown's Calculus of Indications. In: Proc. of the First Conference on Cyberspace, Austin. Schneiderman, B. (1983). Direct Manipulation: A Step Beyond Programming Languages, IEEE Computer, August.

Correspondence and offprint requests to: Gianni Degli Antoni, Universitá degli Studi di Milano, Dipartimento di Scienze della Informazione, Via Moretto da Brescia 9, 20133 Milan, Italy. 